TITLE:

\title{
Swelling and stress-relaxation of poly(N-isopropylacrylamide) gels in the collapsed state
}

\author{
$\operatorname{AUTHOR}(\mathrm{S})$ : \\ Takigawa, T; Ikeda, T; Takakura, Y; Masuda, T
}

\section{CITATION:}

Takigawa, $T$... [et al]. Swelling and stress-relaxation of poly(N-isopropylacrylamide) gels in the collapsed state. JOURNAL OF CHEMICAL PHYSICS 2002, 117(15): 7306-7312

\section{ISSUE DATE:}

2002-10-15

URL:

http://hdl.handle.net/2433/39743

\section{RIGHT:}

Copyright 2002 American Institute of Physics. This article may be downloaded for personal use only. Any other use requires prior permission of the author and the American Institute of Physics. 


\title{
Swelling and stress-relaxation of poly( $\mathrm{N}$-isopropylacrylamide) gels in the collapsed state
}

\author{
Toshikazu Takigawa, ${ }^{\text {a) }}$ Takuji Ikeda, Yoshihito Takakura, and Toshiro Masuda \\ Department of Material Chemistry, Graduate School of Engineering, Kyoto University Sakyo-ku, \\ Kyoto 606-8501, Japan
}

(Received 9 May 2002; accepted 24 July 2002)

\begin{abstract}
Swelling and stress-relaxation under tension for poly(N-isopropylacrylamide) (PNIPA) hydrogels have been investigated in water and in liquid paraffin at various temperatures. Initial Young's modulus is much enhanced due to the change from the swollen to collapsed state, but no difference has been observed in the initial and osmotic Poisson ratios for the gels not only in the collapsed but also in the swollen states. The enhancement of modulus originates from the introduction of physical crosslinks in the collapsed state. In liquid paraffin, the gels in the collapsed phase show large degree of stress reduction in the course of stress-relaxation due to the breakdown of the physical crosslinks. The stress-relaxation induced by swelling is observed for both phases in water, but the degree of stress reduction is larger in the collapsed phase than in the swollen phase. A comparison between experimental results and theory applicable to swelling and swelling-induced stressrelaxation is made to show that the agreement is not so good. For the stress-relaxation of the collapsed gel in water, it is suggested that the swelling couples with the breakdown of physical crosslinks at short times, and the trance of the coupling is observed even at long times. (C) 2002 American Institute of Physics. [DOI: 10.1063/1.1507103]
\end{abstract}

\section{INTRODUCTION}

Poly(N-isopropylacrylamide) (PNIPA) hydrogels have been used to investigate nature of the volume phase transition of polymer gels. ${ }^{1-5}$ The volume phase transition of the PNIPA gels is induced by an infinitesimal change of temperature, and a swollen state emerges at temperatures lower than the transition temperature of ca. $35^{\circ} \mathrm{C}$ and a collapsed phase takes place above the transition temperature. Polymer network chains in the swollen state are solvated by the hydrophobic hydration, 6,7 and thus are very flexible, giving the gels with rubberlike behavior. In the collapsed state the gels contain solvent inside, but the amount of solvent is very low. The network chains in the collapsed state are believed to be solvated with water molecules by hydrogen bonding. The volume phase transition of PNIPA gels originates from the change of solvation mechanism around the transition temperature. The collapsed phase may show different behavior from the swollen state due to its high polymer content, but how the collapsed phase really behaves is still unclear because of lack of information on the structure and properties of the collapsed phase. The original theory of the volume phase transition of polymer gels proposed by Tanaka et al. ${ }^{8}$ is based on the Flory-type free energy. The theory can explain the volume phase transition of charged polymer gels, but cannot be applied to PNIPA gels because they are basically neutral. A new theory has been required to describe the volume phase transition of PNIPA gels, and to build up the theory, structure and properties of the collapsed state must also be clarified.

In the previous papers, ${ }^{9,10}$ we have shown swelling be-

${ }^{a)}$ Electronic mail: takigawa @rheogate.polym.kyoto-u.ac.jp havior of poly(acrylamide) (PAAm) hydrogels under tension. The stress relaxation induced by the swelling under tension, a kind of coupling between mechanical stress and swelling (or, diffusion), was observed for the gels. ${ }^{9}$ When a constant strain is applied to the gels, the gels slowly re-swell to an equilibrium volume, and the applied stress (or, force) decreases with almost the same rate as the volume change to an equilibrium value. A simple theory describing the swellinginduced stress-relaxation has also been proposed. ${ }^{9,10}$ How mechanical stress couples with diffusion gives information on the structure, and therodynamical and mechanical properties of polymer gels. In this study, we investigate swelling and stress-relaxation behavior of PNIPA gels in the collapsed state as well as in the swollen state. The results obtained are compared with the theory ${ }^{9,10}$ summarized in Sec. II. A new type of stress-relaxation occurring in the collapsed phase is modeled in terms of a rate equation.

\section{THEORETICAL BACKGROUND}

The change of the Gibbs free energy $(\Delta F)$ of an isotropic, cubic polymer gel, with unit volume before deformation (namely, in the reference state), under an applied tension $(f)$ can be given by ${ }^{11}$

$$
\begin{aligned}
\Delta F= & N_{s} k_{B} T[\ln (1-\phi)+\chi \phi] \\
& +\frac{N_{c} k_{B} T}{2}\left[\alpha_{\|}^{2}+2 \alpha_{\perp}^{2}-3-\ln \alpha_{\|} \alpha_{\perp}^{2}\right]-f\left(\alpha_{\|}-1\right) .
\end{aligned}
$$

Here, $N_{s}$ is the number of solvent molecules, and $k_{B}, T$, and $\phi$ are, respectively, the Boltzmann constant, the absolute 
temperature, and the polymer volume fraction, $\chi$ being the polymer-solvent interaction parameter. The quantity $N_{c}$ is the number of active chains in the gel, and $\alpha_{\|}$and $\alpha_{\perp}$ are the stretch ratios parallel and normal to the applied tension, respectively. From Eq. (1) swelling (or osmotic) stresses parallel and normal to the tension $\left(\Pi_{\|}\right.$and $\Pi_{\perp}$, respectively) can be defined by ${ }^{9}$

$$
\begin{aligned}
\Pi_{\|} \equiv & \frac{1 \partial \Delta F}{\alpha_{\perp}^{2} \partial \alpha_{\|}} \\
= & \frac{k_{B} T}{\nu_{S}}\left[\ln (1-\phi)+\phi+\chi \phi^{2}\right] \\
& +\frac{N_{c} k_{B} T}{2 \alpha_{\perp}^{2}}\left(2 \alpha_{\|}-\frac{1}{\alpha_{\|}}\right)-\frac{f}{\alpha_{\perp}^{2}} \\
\Pi_{\perp} \equiv & \frac{1}{2 \alpha_{\|} \alpha_{\perp} \partial \alpha_{\perp}} \\
= & \frac{k_{B} T}{\nu_{S}}\left[\ln (1-\phi)+\phi+\chi \phi^{2}\right]+\frac{N_{c} k_{B} T}{2 \alpha_{\|} \alpha_{\perp}}\left(2 \alpha_{\perp}-\frac{1}{\alpha_{\perp}}\right)
\end{aligned}
$$

with $\nu_{s}=(1-\phi) \alpha_{\|} \alpha_{\perp}^{2} / N_{s}$, and these stresses must be zero at equilibrium. Suppose that both $\phi$ and $f$ are small enough, linearized equations for Eq. (2) give to the osmotic (or, equilibrium) Poisson ratio ( $\mu_{\infty}$ ) of $1 / 6$ and the equilibrium stress $\left(\sigma_{\infty}, \sigma_{\infty} \equiv f / \alpha_{\perp}^{2}\right.$ ) of $7 G \epsilon_{\|} / 3$. Here, $G$ is the shear modulus given by $G=N_{c} k_{B} T$, and $\epsilon_{\|}$is the strain parallel to the tension and is related to $\alpha_{\|}$as $\epsilon_{\|}=\alpha_{\|}-1$.

To describe swelling kinetics of the gel under tension, constitutive equations as well as the equation of motion are required. These equations are applied to a small volume element in the gel specimen. Both of the total stress and strain tensors for the volume element ( $\mathbf{s}$ and $\mathbf{u}$, respectively) can be divided into two components: ${ }^{10}$

$$
\mathbf{s}=\mathbf{s}_{\mathrm{os}}+\mathbf{s}_{e}, \quad \mathbf{u}=\mathbf{u}_{\mathrm{os}}+\mathbf{u}_{e} .
$$

Here, $\mathbf{s}_{e}$ and $\mathbf{u}_{e}$ are, respectively, the external stress and strain corresponding to the applied tension, and $\mathbf{s}_{\mathrm{os}}$ and $\mathbf{u}_{\mathrm{os}}$ are the osmotic stress and strain, respectively, which are induced due to the free energy change by the applied tension. When the gel is a linear elastic body, $\mathbf{s}_{\mathrm{os}}$ is related to $\mathbf{u}_{\mathrm{os}}$ as $^{8,10}$

$$
\mathbf{s}_{\mathrm{os}}=2 G\left[\mathbf{u}_{\mathrm{os}}-\frac{1}{3}\left(\operatorname{Tr} \mathbf{u}_{\mathrm{os}}\right) \mathbf{I}\right]+K_{\mathrm{os}}\left[\operatorname{Tr}\left(\mathbf{u}_{\mathrm{os}}-\mathbf{u}_{\mathrm{os}, \infty}\right)\right] \mathbf{I},
$$

where $\mathbf{I}$ is the unit tensor, and $K_{\mathrm{os}}$ is the osmotic bulk modulus related to $\mu_{\infty}$ and $G$ as $K_{\mathrm{os}}=2\left(1+\mu_{\infty}\right) G / 3\left(1-2 \mu_{\infty}\right)$, $\mathbf{u}_{\mathrm{os}, \infty}$ being $\mathbf{u}_{\mathrm{os}}$ at the long time limit. On the other hand, $\mathbf{s}_{e}$ and $\mathbf{u}_{e}$ are related with each other as ${ }^{10}$

$$
\mathbf{s}_{e}=2 G \mathbf{u}_{e}-p \mathbf{I}
$$

if Poisson's ratio as a material constant (initial Poisson's ratio, $\left.\mu_{0}\right)$ is $1 / 2$. The quantity $p$ is a constant determined by a boundary condition. The quantities $G$ and $\mu_{0}$ remain constant in the course of swelling under tension and are identical to those without swelling (e.g., the measured values in air). Because polymer gels actually have $\mu_{0}$ close to $1 / 2$, the value at the incompressible limit, Eq. (5) is applicable as a constitutive equation to polymer gels. The equation of motion for the element in the course of swelling under tension is given by 8,10

$$
\zeta \frac{d \mathbf{v}_{\mathrm{os}}}{d t}=\nabla \mathbf{s}_{\mathrm{os}} .
$$

Here, $\zeta$ is the friction coefficient between the polymer network and solvent molecules, and $\mathbf{v}_{\mathrm{os}}$ is the displace vector corresponding to the osmotic strain $\mathbf{u}_{\mathrm{os}}$. From Eqs. (4) and (6), the time $(t)$ evolution of volume of the element can be described by ${ }^{9,10}$

$$
\begin{aligned}
& \frac{\partial}{\partial t} \operatorname{Tr} \mathbf{u}_{\mathrm{os}}=D_{L} \boldsymbol{\nabla}^{2} \operatorname{Tr} \mathbf{u}_{\mathrm{os}}, \\
& \frac{\partial}{\partial t}\left(\boldsymbol{\nabla} \times \mathbf{v}_{\mathrm{os}}\right)=D_{T} \boldsymbol{\nabla}^{2}\left(\boldsymbol{\nabla} \times \mathbf{v}_{\mathrm{os}}\right)
\end{aligned}
$$

with

$$
D_{L}=\frac{K_{\mathrm{os}}+(4 / 3) G}{\zeta}, \quad D_{T}=\frac{G}{\zeta} .
$$

Equation (7a) holds for $\mathbf{u}$ as well as $\mathbf{u}_{\mathrm{os}}$, as long as $\mu_{0}$ $=1 / 2$ because $\operatorname{Tr} \mathbf{u}_{e}=0$ in this case. For the swelling of a long rectangular gel with a square cross section in the course of stress relaxation, the change of volume actually corresponds to the change in the cross-sectional area $(S)$ and $S$ obeys the following equation: ${ }^{9}$

$$
\begin{aligned}
S= & S_{0}\left\{1-\frac{128}{3 \pi^{4}}\left[\sum_{m, n}^{\text {odd }} \frac{1}{m^{2} n^{2}} \exp \left(-k_{m n} t\right)+\mu_{\infty}\right] \epsilon_{\|}\right\} \\
& \left(\mu_{0}=1 / 2, \mu_{\infty}=1 / 6\right) .
\end{aligned}
$$

Here,

$$
k_{m n}=\frac{m^{2}+n^{2}}{2 \tau_{L}}=\frac{D_{L} \pi^{2}\left(m^{2}+n^{2}\right)}{a_{0}^{2}}
$$

and $S_{0}\left(=a_{0}^{2}\right)$ is the cross-sectional area of the gel in the reference state, and $m$ and $n$ are odd integers. Equation (7b) for the gel in the course of stress relaxation leads to ${ }^{10}$

$$
\frac{\partial}{\partial t} u_{\mathrm{os}, \|}=D_{T} \nabla^{2} u_{\mathrm{os}, \|}
$$

where $u_{\mathrm{os}, \|}$ is the component of $\mathbf{u}_{\mathrm{os}}$ parallel to tension. The global stress $\sigma$ is given from the combination of Eq. (3b) and the solution of Eq. (10) as ${ }^{10}$

$$
\sigma(t)=\frac{G \epsilon_{\|}}{3}\left[7+\frac{128}{\pi^{4}} \sum_{m, n}^{\text {odd }} \frac{1}{m^{2} n^{2}} \exp \left(-k_{m n}^{\prime} t\right)\right]
$$

with

$$
k_{m n}^{\prime}=\frac{m^{2}+n^{2}}{2 \tau_{T}}=\frac{D_{T} \pi^{2}\left(m^{2}+n^{2}\right)}{a_{0}^{2}} .
$$

Equation (11) is based on the assumption that $\sigma_{\infty}=2(1$ $\left.+\mu_{\infty}\right) G \epsilon_{\|}=(7 / 3) G \epsilon_{\|}\left(\mu_{\infty}=1 / 6\right)$ and the initial stress $\left(\sigma_{0}\right)$ is given by $\sigma_{0}=2\left(1+\mu_{0}\right) G \epsilon_{\|}=3 G \epsilon_{\|}\left(\mu_{0}=1 / 2\right)$. It is also 


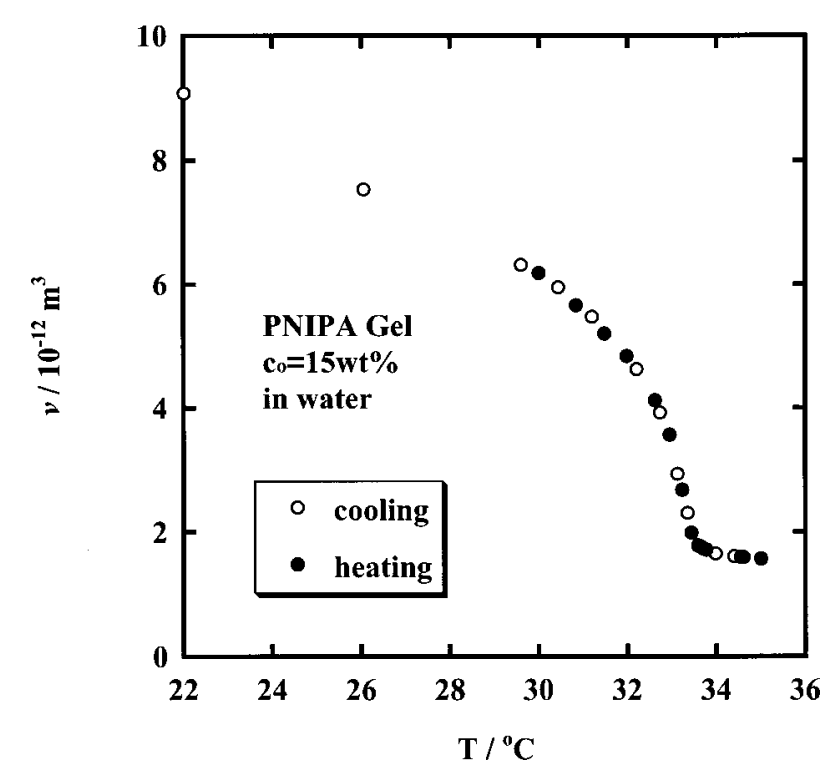

FIG. 1. Temperature dependence of equilibrium volume $(\nu)$ of the cylindrical PNIPA gel.

noted that the ratio of $\tau_{L}$ to $\tau_{T}$ is given by $\tau_{L} / \tau_{T}=D_{T} / D_{L}$ $=2 / 5$ for $\mu_{\infty}=1 / 6$.

\section{EXPERIMENT}

PNIPA gels were prepared by radical copolymerization of $\mathrm{N}$-isopropylacrylamide (NIPA) and $\mathrm{N}, \mathrm{N}^{\prime}$-methylenebis(acrylamide (BIS) in distilled water. Ammonium peroxodisulfate (AP) was used as an initiator, and $\mathrm{N}, \mathrm{N}, \mathrm{N}^{\prime}$, $\mathrm{N}^{\prime}$-tetramethylethylenediamine (TEMED) as an accelerator. The total monomer concentration $\left(c_{0}\right)$ was settled to be 15 wt. \%; $0.153 \mathrm{~mol}$ of NIPA, $2.54 \times 10^{-3} \mathrm{~mol}$ of BIS, 6.13 $\times 10^{-4} \mathrm{~mol}$ of AP, and $240 \mu \mathrm{l}$ of TEMED were dissolved in $100 \mathrm{~g}$ of distilled water. The pregel solution was poured into a metal mold to prepare rectangular gels for mechanical tests, and the gelation was performed at $4{ }^{\circ} \mathrm{C}$ for $24 \mathrm{~h}$. After gelation, the gels were removed from the mold, and were maintained in water at room temperature for 3-4 days and then in water at $65^{\circ} \mathrm{C}$ for $2-3$ weeks to remove residual agents. The cross section of the gel specimens was square in shape and the area was ca. $3.5 \mathrm{~mm} \times 3.5 \mathrm{~mm}$ in the collapsed phase at the temperature equal to or higher than $40^{\circ} \mathrm{C}$ and ca. 6 $\mathrm{mm} \times 6 \mathrm{~mm}$ in the swollen phase at $30^{\circ} \mathrm{C}$.

Stress-strain and stress-relaxation behavior under uniaxial elongation was examined by using an Orientec RTM-500 tensile tester with a liquid bath. The filling solvent in the bath was water or liquid paraffin (nonsolvent for PNIPA), and the temperature of the solvent was controlled within $\pm 0.5^{\circ} \mathrm{C}$. Liquid paraffin was used to investigate stress-relaxation behavior without re-swelling by the applied tension. The initial length for elongation was 0.5 to $1.0 \mathrm{~mm}$, and the crosshead speed was $0.5 \mathrm{~mm} / \mathrm{min}$. The magnitude of imposed strain for stress-relaxation experiments was ca. 0.1. The change of width and length between two marked points parallel to elongation were monitored by the combination of a CCD camera and a TV monitor. Initial Poisson's ration $\mu_{0}$ was determined by the slope of the best fit line to the plot of $-\ln \alpha_{\perp}$ versus $\ln \alpha_{\|}$.

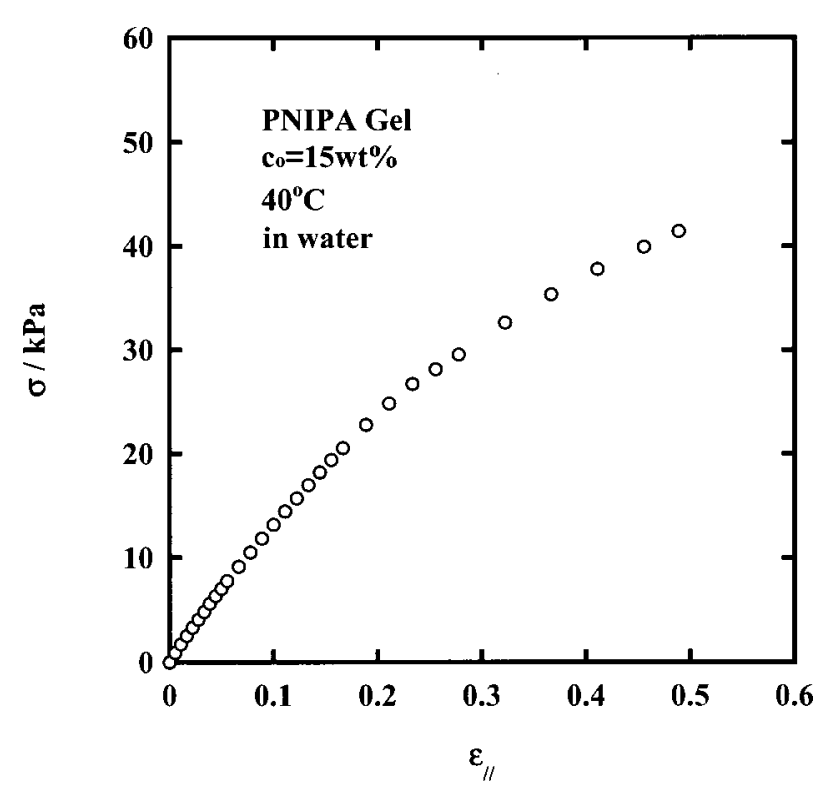

FIG. 2. Stress $(\sigma)$ plotted against strain $\left(\epsilon_{\|}\right)$for the PNIPA gel in water at $40{ }^{\circ} \mathrm{C}$.

\section{RESULTS}

The phase behavior of the PNIPA gel in water was preliminarily investigated. Figure 1 shows the temperature $(T)$ dependence of the equilibrium volume of the PNIPA gel prepared in a thin glass tube in diameter of about $200 \mu \mathrm{m}$. The gel had the same monomer composition and concentration as those used for the mechanical measurements, and the volume in the figure $(\nu)$ was calculated by using the values of diameter and distance between two marked points in the axial direction. The volume of the gel decreases with increasing $T$, and the curve on heating agrees well with that on cooling. No discontinuity is also observed on the curves, indicating that the gel does not undergo the (discontinuous) volume phase transition. Due to the continuous change of volume, a phase boundary between the swollen and collapsed phases cannot be defined exactly for this gel, but in this paper we call the $T$ region higher than $\sim 34^{\circ} \mathrm{C}$ the collapsed phase, and the region lower than that temperature, the swollen phase.

Figure 2 shows the stress $(\sigma)$-strain $\left(\epsilon_{\|}\right)$curve of the PNIPA gel in water at $40^{\circ} \mathrm{C}$. The maximum strain $\left(\epsilon_{\max }\right)$ applied to the specimen was 0.5 . At low strains a linear relation exists between $\sigma$ and $\epsilon_{\|}$, but a downward deviation occurs for $\sigma$ at large strains. Similar behavior was observed commonly for the collapsed gels at the other $T$ in water as well as in liquid paraffin. For the gels in the swollen phase at $30^{\circ} \mathrm{C}$ (both in water and liquid paraffin), only a linear region was observed on $\sigma-\epsilon_{\|}$curves because the specimens failed at relatively low strains. Initial Young's modulus $\left(E_{0}\right)$ for PNIPA gels estimated from the initial slope of $\sigma-\epsilon_{\|}$curves for $\epsilon_{\max }=0.1$ is listed in Table I. The values of $E_{0}$ are independent of $T$ as well as of the type of outside solvent in the collapsed phase, and the values in the swollen phase (at $30{ }^{\circ} \mathrm{C}$ ) are also independent of the type of outside solvent. Figure 3 shows a plot of $-\ln \alpha_{\perp}$ against $\ln \alpha_{\|}$for the gel in water at $40{ }^{\circ} \mathrm{C}$ in the course of elongation of $\epsilon_{\max }=0.1$. Although the data points are a little scattered, a linear relation 
TABLE I. Initial Young's modulus $\left(E_{0}\right)$, initial Poisson's ratio $\left(\mu_{0}\right)$, osmotic Poison's ratio $\left(\mu_{\infty}\right)$, relaxation time determined by volume change $\left(\tau_{V}\right)$, degree of stress reduction $\left(r_{s}\right)$, and relaxation time determined by stress-relaxation $\left(\tau_{\sigma}\right)$ for PNIPA gels at various temperatures.

\begin{tabular}{llccccccc}
\hline \hline Solvent & Phase & $T /{ }^{\circ} \mathrm{C}$ & $E_{0} / 10^{5} \mathrm{~Pa}$ & $\mu_{0}$ & $\mu_{\infty}$ & $\tau_{V} / 10^{4} \mathrm{~s}$ & $r_{s}$ & $\tau_{\sigma} / 10^{4} \mathrm{~s}$ \\
\hline Paraffin & Swollen & 30 & 0.063 & $0.45_{0} \pm 0.01$ & & & 0.13 & $0.9 \pm 0.05$ \\
& Collapsed & 40 & 1.5 & $0.41_{7} \pm 0.02$ & & & 0.30 & $2.6 \pm 0.5$ \\
& Wollapsed & 60 & 1.6 & $0.45_{4} \pm 0.01$ & & & 0.29 & $1.9 \pm 0.2$ \\
\multirow{6}{*}{ Water } & Swollen & 30 & 0.060 & $0.43_{3} \pm 0.02$ & $0.24_{6} \pm 0.01$ & $4.0 \pm 0.3$ & 0.25 & $6.1 \pm 2.0$ \\
& Collapsed & 40 & 1.5 & $0.43_{0} \pm 0.01$ & $0.26_{6} \pm 0.01$ & $1.3 \pm 0.5$ & 0.53 & $3.1 \pm 0.2$ \\
& Collapsed & 45 & 1.6 & $0.45_{6} \pm 0.01$ & $0.20_{6} \pm 0.01$ & $1.3 \pm 0.4$ & 0.58 & $3.6 \pm 0.3$ \\
& Collapsed & 50 & 1.5 & $0.46_{7} \pm 0.01$ & $0.22_{2} \pm 0.01$ & $1.3 \pm 0.4$ & 0.46 & $3.3 \pm 0.2$ \\
& Collapsed & 55 & 1.7 & $0.44_{5} \pm 0.01$ & $0.24_{4} \pm 0.01$ & $0.9 \pm 0.3$ & 0.55 & $2.6 \pm 0.2$ \\
& Collapsed & 60 & 1.7 & $0.43_{0} \pm 0.02$ & $0.22_{7} \pm 0.01$ & $0.8 \pm 0.2$ & 0.49 & $2.6 \pm 0.3$ \\
\hline \hline
\end{tabular}

is observed for the data points, and the slope of the best fit line gives $\mu_{0}=0.430$. The values of $\mu_{0}$ at various temperatures are summarized again in Table I. The values remain almost constant, lying between 0.4 and 0.5 , for all specimens examined, indicating that $\mu_{0}$ is affected neither by the type of phase nor by the type of surrounding solvent.

In Fig. 4 the $t$ dependence of $\epsilon_{\perp}$ after 10\% elongation for the PNIPA gel in water at $30^{\circ} \mathrm{C}$ is shown. This clearly demonstrates that the gel starts to swell by the application of tension. The volume increases very steeply at short times and reaches a constant value around $t=1 \times 10^{5} \mathrm{~s}$. The equilibrium value of $\epsilon_{\perp}\left(\epsilon_{\perp, \infty}\right)$ for this gel leads $\mu_{\infty}\left(\equiv-\epsilon_{\perp, \infty} / \epsilon_{\max }\right)=0.246$, which is much smaller than the corresponding value of $\mu_{0}(0.433)$. The similar plot for the gel in water at $40^{\circ} \mathrm{C}$ is shown in Fig. 5. Even in the collapsed state, the gel shows the re-swelling due to the applied tension, and $\mu_{\infty}$ for this gel specimen (0.266) is close to the value in the swollen state at $30^{\circ} \mathrm{C}(0.246)$. The values of $\mu_{\infty}$ at the other temperatures are tabulated in Table I, together with the above data. The values of $\mu_{\infty}$ are almost constant over the whole $T$ range examined. The longest relaxation time determined from the swelling curve $\left(\tau_{V}\right)$, which corresponds to $\tau_{L}$ in Eq. (8), at various $T$ are also summarized in

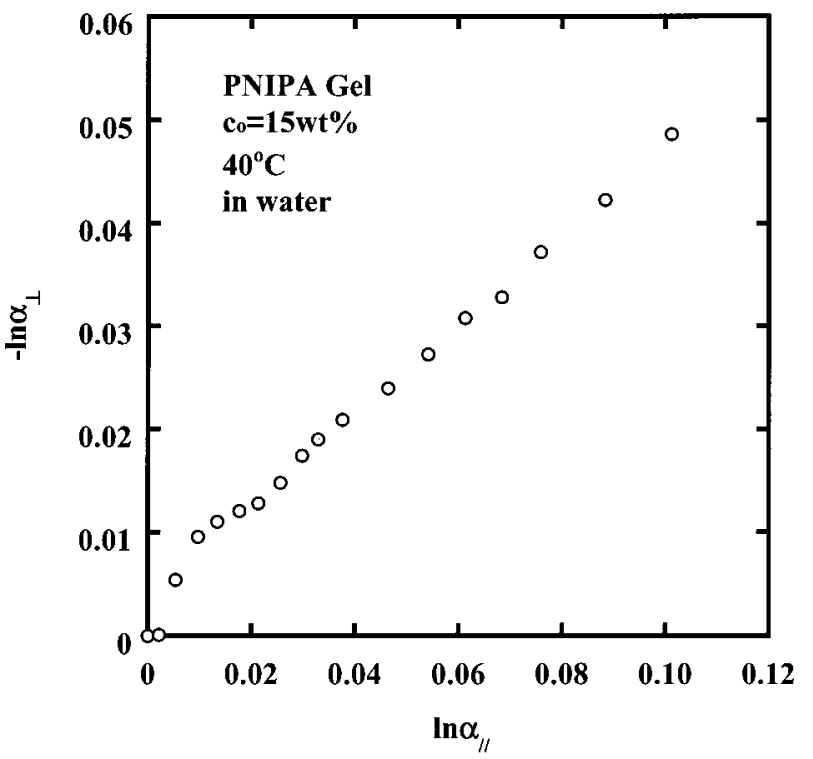

FIG. 3. A plot of $-\ln \alpha_{\perp}$ against $\ln \alpha_{\|}$for the PNIPA gel in water at $40{ }^{\circ} \mathrm{C}$.
Table I. The values of $\tau_{V}$ in the collapsed phase appear to be weakly dependent on $T$, and are much smaller than that in the swollen state. The gels in liquid paraffin re-swelled neither in the swollen phase nor in the collapsed phase.

Figure 6 shows the $T$ dependence of $\sigma$ after $10 \%$ elongation in the swollen state at $30^{\circ} \mathrm{C}$. In water [Fig. 6(a)], the stress decreases monotonically with increasing $t$ and reaches an equilibrium value at about $2 \times 10^{5} \mathrm{~s}$, while the equilibration of stress occurs in a much shorter time scale in liquid paraffin [Fig. 6(b)]. The degree of stress reduction $\left(r_{s}\right)$ is defined by using the initial stress (the stress just after elongation; $\sigma_{0}$ ) as

$$
r_{s}=\frac{\sigma_{0}-\sigma_{\infty}}{\sigma_{0}} .
$$

The value of $r_{s}$ is listed in Table I together with the longest relaxation time for the stress-relaxation $\left(\tau_{\sigma}\right)$ corresponding to $\tau_{T}$ in Eq. (8). For liquid paraffin $r_{s}$ is 0.13 while that for water is 0.25 . The relaxation time $\tau_{\sigma}$ is much smaller for liquid paraffin than for water. Similar plots for the gels in the collapsed phase at $40^{\circ} \mathrm{C}$ are shown in Fig. 7, and $r_{s}$ and $\tau_{\sigma}$

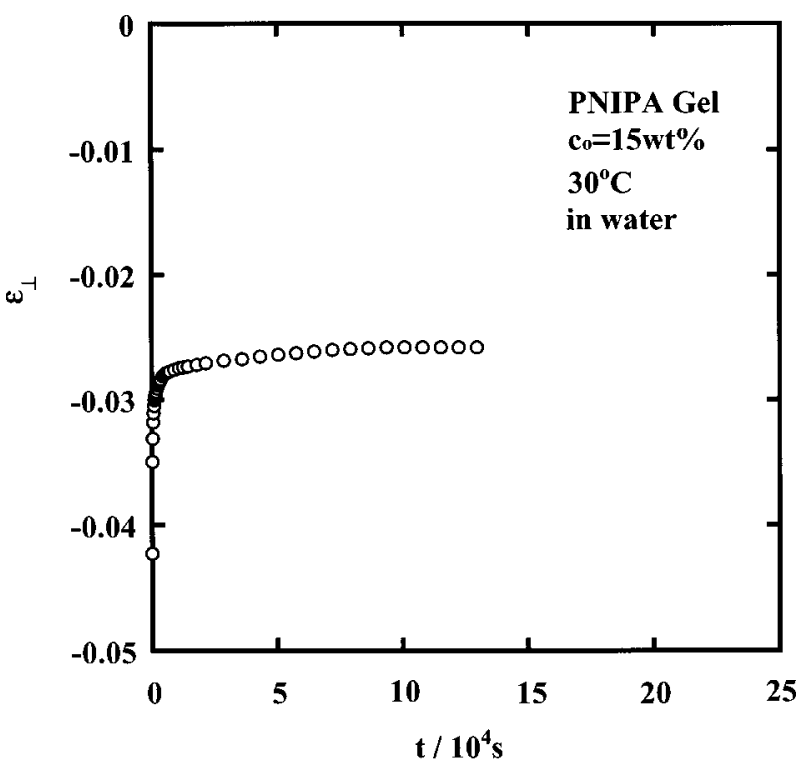

FIG. 4. Time $(t)$ dependence of strain perpendicular to elongation $\left(\epsilon_{\perp}\right)$ for the PNIPA gel at $30^{\circ} \mathrm{C}$. The applied maximum strain for elongation is 0.108 . 


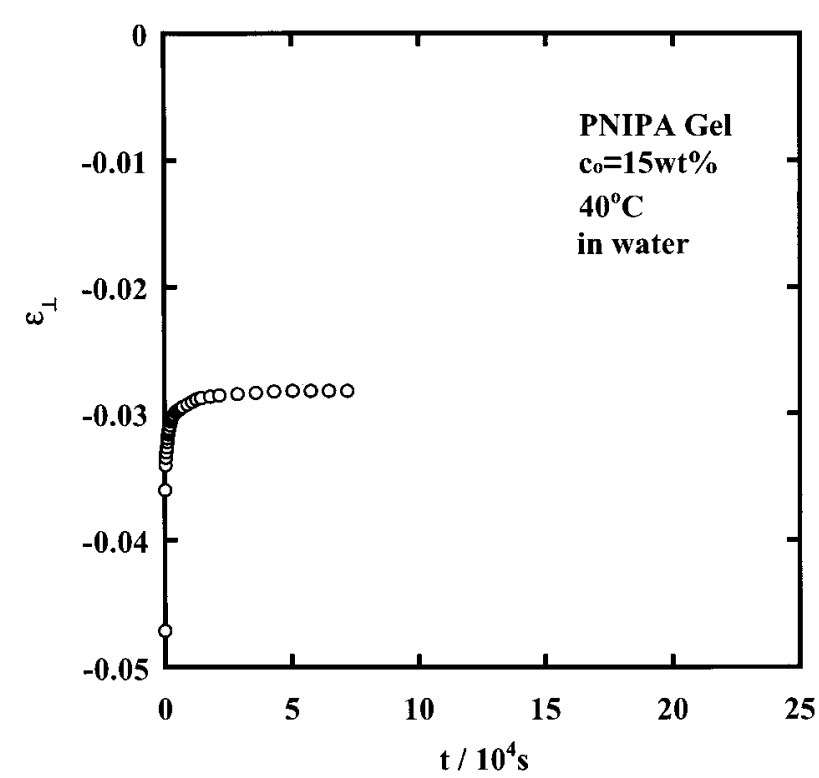

FIG. 5. Time $(t)$ dependence of strain perpendicular to elongation $\left(\epsilon_{\perp}\right)$ for the PNIPA gel at $40{ }^{\circ} \mathrm{C}$. The applied maximum strain for elongation is 0.106 .

are listed in Table I. Although the absolute value of $r_{s}$ at $40{ }^{\circ} \mathrm{C}$ is quite larger than that at $30^{\circ} \mathrm{C}$ both for water and liquid paraffin, there exists a large difference in $r_{s}$ between the two outside solvents at $40^{\circ} \mathrm{C}$. However, the time profile of stress for the two specimens at $40^{\circ} \mathrm{C}$ lies in the same time domain, differently from the gels in the swollen state at $30^{\circ} \mathrm{C}$. The values of $r_{s}$ and $\tau_{\sigma}$ for the other specimens are summarized in Table I. As can be seen from the table, $r_{s}$ is almost independent of $T$ both in water and in liquid paraffin, and the values in liquid paraffin are higher than those in water. Concerning $\tau_{\sigma}$, the two values for the collapsed phase in liquid paraffin are close to each other and are slightly low compared with the values in water.

\section{DISCUSSION}

\section{A. Mechanical properties}

As can be seen from Table I, $E_{0}$ in the collapsed phase is almost constant, resulting from the fact that the volume is almost constant in the collapsed phase above $40^{\circ} \mathrm{C}$. It is very interesting that $E_{0}$ in the collapsed phase is about 25 times higher than the values in the swollen phase. The volume decreases to ca. $1 / 4$ of the volume in the swollen state at $30^{\circ} \mathrm{C}$ (see, Fig. 1), meaning that the polymer concentration (c) becomes 4 times higher by the temperature elevation. As is well known, $E_{0}$ for swollen polymer networks scales with $c$ as ${ }^{12}$

$$
E_{0} \sim c^{1 / 3} \text {. }
$$

This scaling relation indicates that $E_{0}$ in the collapsed phase becomes higher than that at $30{ }^{\circ} \mathrm{C}$, but the calculation based on the above scaling law shows that the degree of enhancement of $E_{0}$ is at highest 2 for the PNIPA gels, while experimental results lead the enhancement of 25 times, meaning that the increase in $E_{0}$ in the collapsed phase dominated by the other mechanism than the change of volume (or $c$ ). This
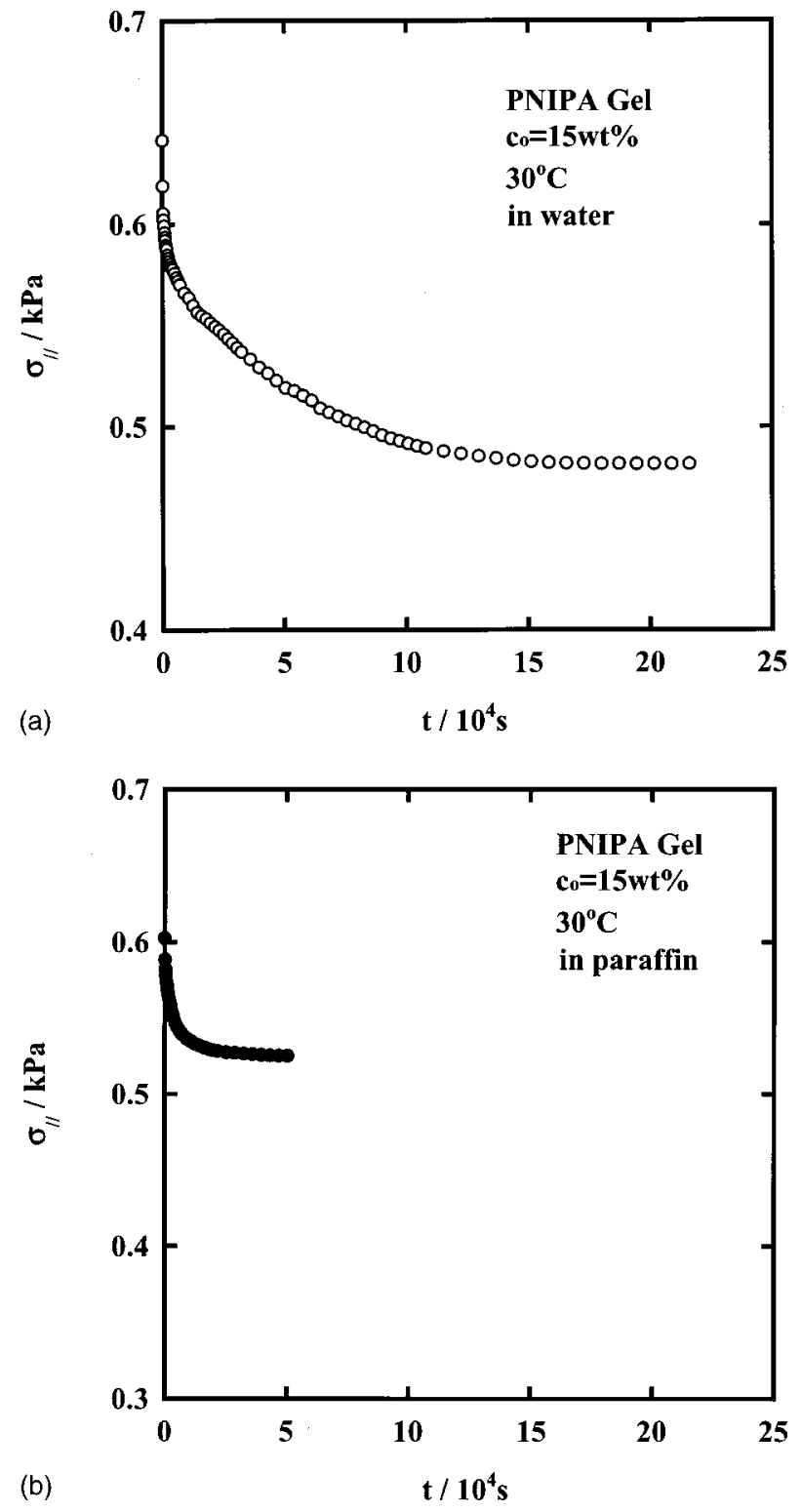

FIG. 6. Plots of stress $(\sigma)$ vs time $(t)$ for PNIPA gels at $30{ }^{\circ} \mathrm{C}$ in water (a) and in liquid paraffin (b). The applied maximum strains for elongation are 0.108 for water and 0.101 for liquid paraffin.

large enhancement of $E_{0}$ must be due to the introduction of physical crosslinks to the gel by the change of solvation from hydrophobic hydration to hydrogen bonding accompanied by the collapse, and the crosslink domain appears to be composed of a sequence or an aggregate of hydrogen bonds. An increase of entanglement density by the collapse, if any, could also enhance the apparent value of $E_{0}$ in the collapsed state. In this case $\sigma$ must decrease to $1 / 25$ of the initial value at equilibrium, but eventually the actual reduction was about $1 / 2$ (see, $r_{s}$ in Table I), suggesting that the enhancement of $E_{0}$ originates from the introduction of physical crosslinks. The fact that $\mu_{0}$ depends neither on the type of phase nor on the type of outside solvent is natural because $\mu_{0}$ is a material constant reflecting only the material (in this case, gel itself) properties. The values of $\mu_{0}$ for the gels are rather close to 


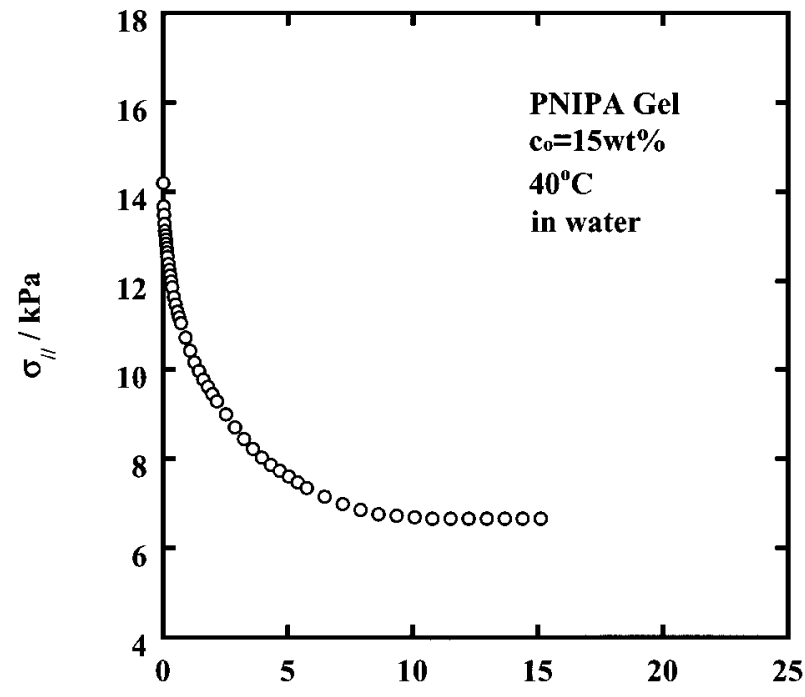

(a)

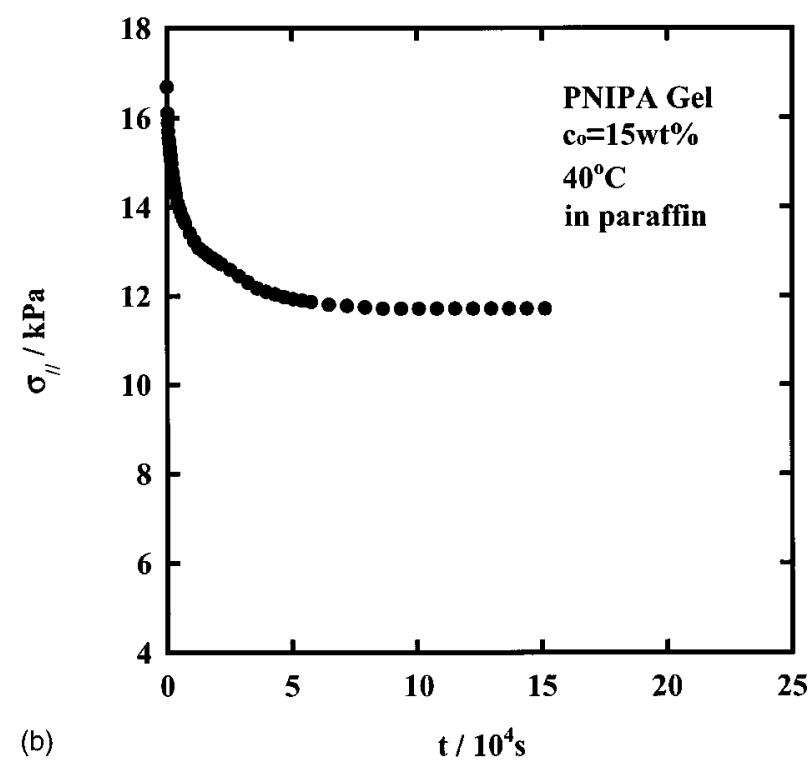

FIG. 7. Plots of stress $(\sigma)$ vs time $(t)$ for PNIPA gels at $40{ }^{\circ} \mathrm{C}$ in water (a) and in liquid paraffin (b). The applied maximum strains for elongation are 0.106 for water and 0.113 for liquid paraffin.

$1 / 2$, the value of the incompressible limit, and agree well with those reported for PAAm gels ${ }^{13}$ and poly(vinyl alcohol) gels. $^{14}$

\section{B. Swelling properties}

The values of $\mu_{\infty}$ in the collapsed state was identical to that in the swollen state and remained constant independently of $T$, suggesting that as long as structural change (such as breakage of crosslinks) does not occur the collapsed phase obeys the same thermodynamics, for example, the Flory-type free energy expression composed of the mixing and rubber elasticity terms is also applicable to the collapsed phase, as the swollen phase, although the breakdown of physical crosslinks by the application of tension actually occurs in the collapsed phase to some extent, as will be discussed later. The network chains in the collapsed phase are flexible as in the case of swollen phase because the theory of rubber elasticity is applicable to the polymer chains in the collapsed phase. The values of $\mu_{\infty}$ estimated from experiment are slightly higher than those for PAAm gels ${ }^{15}$ and also the theoretical prediction (1/6). As stated previously, the theory employed here is a linear one and is based on the assumption that polymer content is low enough, and the assumption is probably still valid for the swollen state but could not be applicable to the collapsed phase because the polymer content in the collapsed phase amounts to $60 \%$. At least for the PNIPA gels in the collapsed state, the enhanced $\mu_{\infty}$ (or, reduced the swelling ratio) might originate from the high polymer content. The values of $\tau_{V}$ in the collapsed state showed a weak $T$ dependence, but we think that the $T$ dependence is only apparent because the standard deviation for $\tau_{V}$ is rather large (Table I), which originates from the difficulty in measurement; the values in the collapsed state must remain constant. The values of $D_{L}$ in the collapsed phase calculated from $\tau_{V}$ and $S_{0}$ are almost comparable with that in the swollen phase, indicating that the enhancement of $E_{0}$, or equivalently $G$, by the increase of $c$ is canceled out by the increase of $\zeta$ in the collapsed phase [Eq. (8)].

\section{Stress-relaxation in liquid paraffin}

The stress-relaxation in liquid paraffin at $30^{\circ} \mathrm{C}$ (in the swollen state) showed a small value of $r_{s}(0.13)$ and the value of $\tau_{\sigma}$ was also very small. Of course, the swelling by tension does not contribute to this stress-relaxation process because the gel does not re-swell in liquid paraffin, suggesting that the mechanism of this stress-relaxation is identical to that for real elastomers, such as entanglement relaxation of dangling chains. In the collapsed phase, the stress-relaxation in liquid paraffin shows quite different behavior: $\tau_{\sigma}$ becomes larger comparable to the values in water and $r_{s}$ becomes much large amounting to 0.3 . In the collapsed phase, the contribution from such as entanglement relaxation to whole relaxation is negligible, and accordingly another mechanism becomes dominant. This must be due to the breakdown of crosslinks by the applied tension. As stated before, physical crosslinks are introduced in the collapsed phase. The applied tension, of course, cannot scissor the chemical crosslinks introduced in the sample preparation stage, but can break down the physical crosslinks. Actually, the downward deviation of the $\sigma-\epsilon_{\|}$curve in Fig. 1 suggests that the physical crosslinks are broken down by the applied tension. The stressrelaxation due to the breakdown of physical crosslinks, as observed for the gel in the collapsed phase in liquid paraffin, can be explained by a simple rate equation, if we assume that the annihilation rate is proportional to an extra stress, $\sigma(t)$ $-\sigma_{\infty}$. Let $n_{c}$ and $n_{p}$ be the number densities of active (i.e., force supporting) chemical and physical crosslinks, respectively. The time dependence of $n_{p}$ is determined by the following equation:

$$
\frac{d n_{p}(t)}{d t}=-k_{d}\left[n_{p}(t)-n_{p}(\infty)\right],
$$

where $k_{d}$ is a rate constant for crosslink annihilation, $n_{p}(\infty)$ being the final value of $n_{p}$, Eq. (14) gives the relaxation modulus $G(t)$, applicable to the long time region, of the form 


$$
\frac{G(t)}{k_{B} T} \approx\left[n_{c}+n_{p}(\infty)\right]+\left[n_{p}(0)-n_{p}(\infty)\right] \exp \left(-k_{d} t\right) .
$$

Here, $n_{p}(0)$ is the initial value of $n_{p}$. The rate constant $k_{d}$ is equal to $1 / \tau_{\sigma}$ for liquid paraffin. Once a physical crosslink is broken in the course of stress-relaxation, the broken fragments will again form a new crosslink. This means the total number of physical crosslinks remains constant independently of $t$, but the new crosslink never participates in stress; the number of active crosslinks decreases monotonically with increasing $t$.

\section{Stress-relaxation in water}

In the swollen state at $30{ }^{\circ} \mathrm{C}$, the gel in water showed larger values of $r_{s}$ and $\tau_{\sigma}$ compared with the specimen in liquid paraffin, indicating that the stress-relaxation in water is strongly controlled by the swelling under tension. The ratio $\tau_{V} / \tau_{\sigma}$ at $30^{\circ} \mathrm{C}$ is 0.66 while the theory in Sec. II gives the value of 0.40 . Although it is unclear at present why the difference occurs between the experimental and theoretical values, PAAm gels have also shown larger values of the ratio close to unity. ${ }^{9}$ The difference in $r_{s}$ between the gels in water and liquid paraffin (0.12), which corresponds to the stress reduction originating from pure swelling, is slightly smaller than the theoretical value of $r_{s}(0.22)$.

In the collapsed phase, the gel in water showed an enhanced stress reduction, larger value of $r_{s}$, compared with the gel in liquid paraffin, indicating the existence of the swelling-induced stress-relaxation even in the collapsed state. The stress-relaxation in the collapsed phase must have three origins: relaxation observed commonly for elastomers such as entanglement relaxation, breakdown of physical crosslinks, and swelling by the application of tension. The values of $r_{s}$ and $\tau_{\sigma}$ in the collapsed phase are scattered (Table I), but both quantities appear to remain almost constant over the $T$ range examined, suggesting that the percentage contribution from each origin to whole relaxation is unchanged over the $T$ region of $40-60{ }^{\circ} \mathrm{C}$. The long-time behavior is controlled by the latter two contributions. In this case, the long-time behavior in stress-relaxation may be described by the combination of Eq. (15) and an asymptotic form of Eq. (11); namely, by the following equation:

$$
\begin{aligned}
\sigma \approx & \frac{k_{B} T \epsilon_{\|}}{3}\left[7+\frac{128}{\pi^{4}} \exp \left(-k_{11}^{\prime} t\right)\right]\left\{\left[n_{c}+n_{p}(\infty)\right]\right. \\
& \left.+\left[n_{p}(0)-n_{p}(\infty)\right] \exp \left(-k_{d} t\right)\right\} .
\end{aligned}
$$

The experimental results for $\tau_{\sigma}$ in Table I indicate that $k_{11}^{\prime}$ $\cong k_{d}$. In the early stage of stress-relaxation of the gels in the collapsed state in water, both breakdown of physical crosslinks and swelling occur simultaneously inside the gels, and the breakdown must accelerate swelling; namely, swelling couples with the breakdown of physical crosslinks. The coupled swelling thus changes the rate of stress-relaxation under tension. The fact that $k_{11}^{\prime} \cong k_{d}$, which is the result in the long-time region, may be the trace of the coupling in the short-time domain. To clarify this point, more extensive and precise experiments will be needed.

\section{CONCLUSIONS}

It was clearly shown from experiment that PNIPA gels in water swell by the application of tension even in the collapsed state, where the polymer content is very high. This must suggest that the collapsed phase has a network structure with flexible polymer chains just as a swollen phase. It was identified that whole stress-relaxation of PNIPA gels is composed of three contributions: relaxation observed commonly for elastomers, breakdown of physical crosslinks, and swelling-induced relaxation. An expression for the relaxation modulus originating from the breakdown of physical crosslinks, applicable to the long-time behavior of stressrelaxation of the PNIPA gels in the collapsed state in liquid paraffin, was presented. The expression was also extended to apply to the long-time stress-relaxation behavior of the collapsed gels in water, where the relaxation due to breakdown of physical crosslinks as well as swelling under tension is dominant. The results on the stress-relaxation of the PNIPA gels in the collapsed phase in water suggested that swelling couples with the breakdown of physical crosslinks in the short-time region, and the coupling affects the long-time behavior of the swelling and swelling-induced stress relaxation. A comparable theory with experimental results on the swelling and swelling-induced stress-relaxation was briefly introduced, and the comparison with experiment was made. The theory was not able to explain the experimental results on the swelling and swelling-induced stress-relaxation of the PNIPA gels in the quantitative level. A more refined model will be required to understand the entire picture of the swelling and stress-relaxation of collapsed PNIPA gels.

${ }^{1}$ Y. Hirokawa, T. Tanaka, and E. S. Matsuo, J. Chem. Phys. 81, 6379 (1984).

${ }^{2}$ S. Horotsu, Y. Hirokawa, and T. Tanaka, J. Chem. Phys. 87, 1392 (1987).

${ }^{3}$ S. Hirotsu, J. Chem. Phys. 88, 427 (1988).

${ }^{4}$ H. Inomata, S. Goto, and S. Saito, Macromolecules 23, 4887 (1990).

${ }^{5}$ M. Tokita and T. Tanaka, J. Chem. Phys. 95, 4613 (1991).

${ }^{6}$ M. Shibayama and T. Tanaka, Adv. Polym. Sci. 109, 1 (1993).

${ }^{7}$ S. Saito, M. Konno, and H. Inomata, Adv. Polym. Sci. 109, 207 (1993).

${ }^{8}$ T. Tanaka, L. O. Hocker, and G. B. Benedek, J. Chem. Phys. 59, 5151 (1973).

${ }^{9}$ T. Takigawa, K. Urayama, Y. Morino, and T. Masuda, Polym. J. (Tokyo) 25, 929 (1993).

${ }^{10}$ T. Takigawa, K. Urayama, and T. Masuda, Polym. Gels Networks 2, 59 (1994).

${ }^{11}$ S. Hirotsu and A. Onuki, J. Phys. Soc. Jpn. 58, 1508 (1989).

${ }^{12}$ L. G. R. Treloar, The Physics of Rubber Elasticity, 2nd ed. (Oxford University Press, London, 1958).

${ }^{13}$ T. Takigawa, Y. Morino, K. Urayama, and T. Masuda, Polym. Gels Networks 4, 1 (1996).

${ }^{14}$ K. Urayama, T. Takigawa, and T. Masuda, Macromolecules 26, 3092 (1993).

${ }^{15}$ T. Takigawa, Y. Morino, K. Urayama, and T. Masuda, Polym. J. (Tokyo) 28, 1012 (1996). 